\title{
Square-full numbers with an even number of prime factors
}

\author{
Rafael Jakimczuk \\ División Matemática, Universidad Nacional de Luján \\ Buenos Aires, Argentina \\ e-mail: jakimczu@mail.unlu.edu.ar
}

Received: 15 June 2019

Revised: 24 February 2020

Accepted: 5 March 2020

\begin{abstract}
In this article, we study the functions $\omega(n)$ and $\Omega(n)$, where $n$ is an $s$-full number. For example, we prove that the square-full numbers with $\Omega(n)$ even are in greater proportion than the square-full numbers with $\Omega(n)$ odd. The methods used are elementary.
\end{abstract}

Keywords: Square-full numbers, Arithmetical functions $\omega(n)$ and $\Omega(n)$.

2010 Mathematics Subject Classification: 11A99, 11B99.

\section{Introduction and preliminary notes}

Let us consider the prime factorization of a positive integer $n=q_{1}^{s_{1}} \cdots q_{r}^{s_{r}}$, where $q_{i}$ $(i=1, \ldots, r)(r \geq 1)$ are the different primes in the prime factorization and $s_{i}(i=1, \ldots, r)$ are the multiplicities or exponents. We need the following well-known arithmetical functions: $\omega(n)=r$ that is the number of different prime factors in the prime factorization of $n$, $\Omega(n)=s_{1}+\cdots+s_{r}$ that is the total number of prime factors in the prime factorization of $n, u(n)=q_{1} \cdots q_{r}$ that denotes the kernel of $n$ and $w(n)=\left(q_{1}+1\right) \cdots\left(q_{r}+1\right)$. Note that $w(n)$ is the sum of the positive divisors of the kernel of $n$.

The functions $\omega(n)$ and $\Omega(n)$ were studied by G. H. Hardy and S. Ramanujan in 1917 [6]. They obtained the following formulas

$$
\sum_{n \leq x} \omega(n)=x \log \log x+M x+o(x),
$$




$$
\sum_{n \leq x} \Omega(n)=x \log \log x+\left(M+\sum_{p} \frac{1}{p(p-1)}\right) x+o(x),
$$

where $M$ is Mertens's constant. In the same paper they define the normal order of an arithmetical function and they prove that the normal order of $\omega(n)$ and $\Omega(n)$ is $\log \log n$.

Let $\Omega_{p}(x)$ be the number of positive integers $n$ not exceeding $x$ such that $\Omega(n)$ is even and $\Omega_{i}(x)$ the number of positive integers $n$ not exceeding $x$ such that $\Omega(n)$ is odd. The following asymptotic formulas are well-known

$$
\Omega_{i}(x)=\frac{1}{2} x+o(x), \quad \Omega_{p}(x)=\frac{1}{2} x+o(x) .
$$

That is, these two sets of positive integers have density $1 / 2$.

Let $\omega_{p}(x)$ be the number of positive integers $n$ not exceeding $x$ such that $\omega(n)$ is even and $\omega_{i}(x)$ is the number of positive integers $n$ not exceeding $x$ such that $\omega(n)$ is odd. Recently, $\mathbf{R}$. Jakimczuk [10] proved that also these two sets of positive integers have density $1 / 2$. That is

$$
\omega_{i}(x)=\frac{1}{2} x+o(x), \quad \omega_{p}(x)=\frac{1}{2} x+o(x) .
$$

A number is $h$-full if all the distinct primes in its prime factorization have multiplicity (or exponent) greater than or equal to $h$. If $h=2$ the numbers are called square-full. The square-full numbers were studied by P. Erdős and G. Szekeres [3] and many other authors. For example, P. T. Bateman and E. Grosswald [1], A. Ivić and P. Shiu (see [8] and [9]), S. W. Golomb [5], etc. Also, recently, R. Jakimczuk [12] studied the kernel of $h$-full numbers. See also the reference [2]. An elementary proof on the distribution of $h$-full numbers is established here.

In this article, we study the functions $\Omega(n)$ and $\omega(n)$ on the $h$-full numbers. In particular, on the square-full numbers. For example, between other results, we prove that the square-full numbers $n$ with $\Omega(n)$ even are in greater proportion than the square-full numbers $n$ with $\Omega(n)$ odd.

We shall need the following theorems on the distribution of square-free numbers. In this note a square-free number will be denoted $q_{1}$.

Theorem 1.1. Let $Q_{1}(x)$ be the number of square-free numbers not exceeding $x$, we have

$$
Q_{1}(x)=\sum_{q_{1} \leq x} 1=\frac{6}{\pi^{2}} x+o(x) .
$$

Let $Q_{p}(x)$ be the number of square-free n not exceeding $x$ such that $\Omega(n)=\omega(n)$ is even and let $Q_{i}(x)$ be the number of square-free $n$ not exceeding $x$ such that $\Omega(n)=\omega(n)$ is odd. We have (prime number theorem)

$$
\begin{aligned}
& Q_{p}(x)=\frac{1}{2} \frac{6}{\pi^{2}} x+o(x), \\
& Q_{i}(x)=\frac{1}{2} \frac{6}{\pi^{2}} x+o(x) .
\end{aligned}
$$

Proof. See [7, chapter XVIII]. 
In this note a square-free multiple of the different and fixed primes $q_{1}, \ldots, q_{s}$, that is multiple of the square-free $q_{1} q_{2} \cdots q_{s}$, will be denoted $q_{q_{1} \cdots q_{s}}$.

Theorem 1.2. Let $Q_{q_{1} \cdots q_{s}}(x)$ be the number of square-free not exceeding $x$ multiple of the different and fixed primes $q_{1}, q_{2}, \ldots, q_{s}$, we have

$$
Q_{q_{1} q_{2} \cdots q_{s}}(x)=\sum_{q_{q_{1} q_{2} \cdots q_{s} \leq x}} 1=\frac{6}{\pi^{2}} \prod_{i=1}^{s} \frac{1}{q_{i}+1} x+o(x) .
$$

Proof. See [11].

Let $(M P)_{q_{1} \cdots q_{s}}(x)$ be the number of square-free $n$ not exceeding $x$ multiple of $q_{1} \cdots q_{s}$ such that $\Omega(n)=\omega(n)$ is even. On the other hand, let $(M I)_{q_{1} \cdots q_{s}}(x)$ be the number of square-free $n$ not exceeding $x$ multiple of $q_{1} \cdots q_{s}$ such that $\Omega(n)=\omega(n)$ is odd. We have the following theorem.

Theorem 1.3. The following asymptotic formulas hold.

$$
\begin{aligned}
& (M P)_{q_{1} \cdots q_{s}}(x)=\frac{1}{2} \frac{6}{\pi^{2}} \prod_{i=1}^{s} \frac{1}{q_{i}+1} x+o(x), \\
& (M I)_{q_{1} \cdots q_{s}}(x)=\frac{1}{2} \frac{6}{\pi^{2}} \prod_{i=1}^{s} \frac{1}{q_{i}+1} x+o(x) .
\end{aligned}
$$

Proof. See [10].

Theorem 1.4. If $\alpha>0$ the following two series of positive terms are convergent

$$
\sum_{n=1}^{\infty} \frac{1}{w(n) n^{\alpha}}, \quad \sum_{n=1}^{\infty} \frac{1}{u(n) n^{\alpha}}
$$

and besides the following two equations hold

$$
\begin{gathered}
\sum_{n=1}^{\infty} \frac{1}{w(n) n^{\alpha}}=\prod_{p}\left(1+\frac{1}{(p+1)\left(p^{\alpha}-1\right)}\right), \\
\sum_{n=1}^{\infty} \frac{1}{u(n) n^{\alpha}}=\prod_{p}\left(1+\frac{1}{p\left(p^{\alpha}-1\right)}\right),
\end{gathered}
$$

where the notation $\prod_{p}$ means that the product runs over all positive primes $p$.

Proof. We have

$$
\begin{aligned}
& \sum_{n=1}^{\infty} \frac{1}{w(n) n^{\alpha}}=\prod_{p}\left(1+\frac{1}{(p+1) p^{\alpha}}+\frac{1}{(p+1)\left(p^{\alpha}\right)^{2}}+\frac{1}{(p+1)\left(p^{\alpha}\right)^{3}}+\cdots\right) \\
= & \prod_{p}\left(1+\frac{1}{(p+1) p^{\alpha}}\left(\frac{1}{1-\frac{1}{p^{\alpha}}}\right)\right)=\prod_{p}\left(1+\frac{1}{(p+1)\left(p^{\alpha}-1\right.}\right) .
\end{aligned}
$$


Now, the product

$$
\prod_{p}\left(1+\frac{1}{(p+1)\left(p^{\alpha}-1\right)}\right)
$$

converges to a positive number, since the series of positive terms

$$
\sum_{p} \frac{1}{(p+1)\left(p^{\alpha}-1\right)}
$$

clearly converges. The theorem is proved.

\section{Main results}

Let $h \geq 2$ be an arbitrary but fixed positive integer. A number is $h$-full if all the distinct primes in its prime factorization have multiplicity (or exponent) greater than or equal to $h$. That is, the number $q_{1}^{s_{1}} \cdots q_{r}^{s_{r}}$ is $h$-full if $s_{i} \geq h(i=1, \ldots, r)(r \geq 1)$. We shall denote a general $h$-full number $n_{h}$. If $h=2$, the numbers are called square-full. The $h$-kernel of the $h$-full number $n_{h}$ we define in the form $\left(u\left(n_{h}\right)\right)^{h}$ and the $h$-remainder in the form $\frac{n_{h}}{\left(u\left(n_{h}\right)\right)^{h}}$. Note that the $h$-remainder is 1 if and only if the $h$-full number is of the form $\left(q_{1} \cdots q_{r}\right)^{h}$.

Let $A_{h}(x)$ be the number of $h$-full numbers not exceeding $x$.

Theorem 2.1. Let $h \geq 2$ be an arbitrary but fixed positive integer. The following asymptotic formula holds

$$
A_{h}(x)=\sum_{n_{h} \leq x} 1=\frac{6}{\pi^{2}} C_{0, h} x^{\frac{1}{h}}+o\left(x^{\frac{1}{h}}\right)
$$

where

$$
C_{0, h}=\sum_{n=1}^{\infty} \frac{1}{w(n)} \frac{1}{n^{\frac{1}{h}}}=\prod_{p}\left(1+\frac{1}{(p+1)\left(p^{\frac{1}{h}}-1\right)}\right) \quad(w(1)=1) .
$$

Proof. Let us consider the prime factorization of a positive integer $a \geq 2$

$$
a=q_{1}^{s_{1}} q_{2}^{s_{2}} \cdots q_{t}^{s_{t}},
$$

where $q_{1}, q_{2}, \ldots, q_{t}$ are the different primes in the prime factorization of $a$. We put

$$
a^{\prime}=q_{1} q_{2} \cdots q_{t}
$$

and

$$
a^{\prime \prime}=\left(q_{1}+1\right)\left(q_{2}+1\right) \cdots\left(q_{t}+1\right) .
$$

If $a=1$, then we put $a^{\prime}=a^{\prime \prime}=1$.

Therefore, we have (see Theorem 1.1 and Theorem 1.2)

$$
\sum_{q_{a^{\prime}} \leq x} 1=\frac{6}{\pi^{2}} \frac{1}{a^{\prime \prime}} x+o(x) \text {. }
$$


Let us consider the set $H$ of all $h$-full numbers $n_{h}$ not exceeding $x$. Now, let us consider the set $T_{a}$ of all $h$-full numbers $n_{h}$ not exceeding $x$ with the same $h$-remainder $a$, that is, $T_{a}=\left\{n_{h}: n_{h} \leq x, v_{h}\left(n_{h}\right)=a\right\}$. Note that if $a_{1} \neq a_{2}$ we have $T_{a_{1}} \cap T_{a_{2}}=\phi$, that is, the sets $T_{a_{1}}$ and $T_{a_{2}}$ are disjoint. Suppose that $A_{x}$ (depending on $x$ ) is the greatest $h$-remainder among the numbers in the set $H$. Then

$$
\bigcup_{a=1}^{A_{x}} T_{a}=H .
$$

Therefore, the sets $T_{a}$ are partitions of the set $H$. Note that some $T_{a}$ can be empty.

The set of the $h$-kernel of the numbers in the set $T_{a}$ will be denoted by $S_{a}$. Hence,

$$
S_{a}=\left\{q_{a^{\prime}}^{h}: q_{a^{\prime}}^{h} \leq \frac{x}{a}\right\}=\left\{q_{a^{\prime}}^{h}: q_{a^{\prime}} \leq \frac{x^{(1 / h)}}{a^{(1 / h)}}\right\} .
$$

The series $\sum_{a=1}^{\infty} \frac{1}{a^{\prime \prime}} \frac{1}{a^{\frac{1}{h}}}$ converges (see Theorem 1.4). Hence

$$
\sum_{a=1}^{\infty} \frac{1}{a^{\prime \prime}} \frac{1}{a^{\frac{1}{h}}}=C_{0, h} .
$$

We choose $B$ such that (see Theorem 1.4)

$$
\sum_{a=B+1}^{\infty} \frac{1}{a^{\prime \prime}} \frac{1}{a^{\frac{1}{h}}}<\epsilon
$$

and

$$
\frac{\pi^{2}}{6} \sum_{a=B+1}^{\infty} \frac{1}{a^{\prime} a^{\frac{1}{h}}}<\epsilon .
$$

Therefore, we have (see (3), (4), (5) and (6))

$$
\begin{aligned}
& A_{h}(x)=\sum_{a=1}^{A(x)}\left(\sum_{q_{a^{\prime}} \leq \frac{x^{(1 / h)}}{a^{(1 / h)}}} 1\right)=\sum_{a=1}^{B}\left(\sum_{q_{a^{\prime}} \leq \frac{x^{(1 / h)}}{a^{(1 / h)}}} 1\right)=\sum_{a=1}^{B}\left(\frac{1}{a^{\prime \prime}} \frac{6}{\pi^{2}} \frac{x^{\frac{1}{h}}}{a^{\frac{1}{h}}}\right)+o\left(x^{\frac{1}{h}}\right) \\
& +\sum_{a=B+1}^{A(x)}\left(\sum_{q_{a^{\prime}} \leq \frac{x^{(1 / h)}}{a^{(1 / h)}}} 1\right)=\frac{6}{\pi^{2}} x^{\frac{1}{h}}\left(\sum_{a=1}^{B} \frac{1}{a^{\prime \prime}} \frac{1}{a^{\frac{1}{h}}}\right)+o\left(x^{\frac{1}{h}}\right) \\
& +\sum_{a=B+1}^{A(x)}\left(\sum_{q_{a^{\prime}} \leq \frac{x^{(1 / h)}}{a^{(1 / h)}}} 1\right)=\frac{6}{\pi^{2}} x^{\frac{1}{h}} C_{0, h}-\frac{6}{\pi^{2}} x^{\frac{1}{h}}\left(\sum_{a=B+1}^{\infty} \frac{1}{a^{\prime \prime}} \frac{1}{a^{\frac{1}{h}}}\right) \\
& +\sum_{a=B+1}^{A(x)}\left(\sum_{q_{a^{\prime}} \leq \frac{x^{(1 / h)}}{a^{(1 / h)}}} 1\right) \\
& +\quad o(1) \frac{6}{\pi^{2}} x_{\frac{1}{h}}+\sum_{a=B+1}^{A(x)}\left(\sum_{q_{a^{\prime}} \leq \frac{x^{(1 / h)}}{a^{(1 / h)}}} 1\right.
\end{aligned}
$$


Equation (8) can be written in the form

$$
\begin{aligned}
& \frac{A_{h}(x)}{\frac{6}{\pi^{2}} x^{\frac{1}{h}}}-C_{0, h}=-\left(\sum_{a=B+1}^{\infty} \frac{1}{a^{\prime \prime}} \frac{1}{a^{\frac{1}{h}}}\right)+o(1) \\
+ & \frac{\sum_{a=B+1}^{A(x)}\left(\sum_{q_{a^{\prime}} \leq \frac{x^{(1 / h)}}{a^{(1 / h)}}} 1\right)}{\frac{6}{\pi^{2}} x^{\frac{1}{h}}} .
\end{aligned}
$$

We have (see (8) and (7))

$$
\begin{aligned}
0 & \leq \sum_{a=B+1}^{A(x)}\left(\sum_{q_{a^{\prime}} \leq \frac{x^{(1 / h)}}{a^{(1 / h)}}} 1\right) \leq \sum_{a=B+1}^{A(x)}\left(\sum_{q_{1} \leq \frac{x^{\prime}(/ h)}{a^{\prime} a^{(1 / h)}}} 1\right) \\
& \leq \sum_{a=B+1}^{A(x)}\left(\sum_{n \leq \frac{x^{(1 / h)}}{a^{\prime} a^{(1 / h)}}} 1\right) \leq \sum_{a=B+1}^{A(x)}\left(\frac{x^{(1 / h)}}{a^{\prime} a^{(1 / h)}}\right) \\
& =x^{\frac{1}{h}} \sum_{a=B+1}^{A(x)} \frac{1}{a^{\prime} a^{\frac{1}{h}}} \leq \frac{6}{\pi^{2}} x^{\frac{1}{h}} \frac{\pi^{2}}{6} \sum_{a=B+1}^{\infty} \frac{1}{a^{\prime} a^{\frac{1}{h}}} \\
& \leq \epsilon \frac{6}{\pi^{2}} x^{\frac{1}{h}} .
\end{aligned}
$$

We choose $x_{0}$ such that if $x \geq x_{0}$ then $|o(1)|<\epsilon$ in equation (9). Equations (9), (6) and (10) give

$$
\left|\frac{A_{h}(x)}{\frac{6}{\pi^{2}} x^{\frac{1}{h}}}-C_{0, h}\right| \leq 3 \epsilon .
$$

Therefore, since $\epsilon$ is arbitrarily small, we have

$$
\lim _{x \rightarrow \infty} \frac{A_{h}(x)}{\frac{6}{\pi^{2}} x^{\frac{1}{h}}}=C_{0, h} .
$$

That is (1). The theorem is proved.

Remark 2.2. If $h=2$ then it is well-known that the constant can be written in terms of the Riemann zeta function $\zeta(s)$, that is, the value of the constant is $\frac{\zeta(3 / 2)}{\zeta(3)}$. This can be obtained from our formulas (16) and (17), since

$$
\begin{aligned}
& \frac{6}{\pi^{2}} C_{0,2}=\prod_{p}\left(\left(1-\frac{1}{p^{2}}\right)\left(1+\frac{1}{(p+1)\left(p^{1 / 2}-1\right)}\right)\right) \\
= & \prod_{p}\left(1-\frac{1}{p^{2}}+\frac{p^{1 / 2}+1}{p^{2}}\right)=\prod_{p}\left(1+\frac{1}{p^{3 / 2}}\right)=\prod_{p}\left(\frac{\frac{1}{1-p^{-3 / 2}}}{\frac{1}{1-p^{-3}}}\right) \\
= & \frac{\zeta(3 / 2)}{\zeta(3)}=2.1732543125 \ldots
\end{aligned}
$$

See [4, page 112]. 
Let $\omega_{p, h}(x)$ be the number of $h$-full numbers $n_{h}$ not exceeding $x$ such that $\omega\left(n_{h}\right)$ is even and let $\omega_{i, h}(x)$ be the number of $h$-full numbers $n_{h}$ not exceeding $x$ such that $\omega\left(n_{h}\right)$ is odd. We have the following theorem.

Theorem 2.3. The following asymptotic formulas hold.

$$
\begin{aligned}
& \omega_{p, h}(x)=\frac{1}{2} \frac{6}{\pi^{2}} C_{0, h} x^{\frac{1}{h}}+o\left(x^{\frac{1}{h}}\right), \\
& \omega_{i, h}(x)=\frac{1}{2} \frac{6}{\pi^{2}} C_{0, h} x^{\frac{1}{h}}+o\left(x^{\frac{1}{h}}\right) .
\end{aligned}
$$

Proof. The proof of (11) is the same as the proof of Theorem 2.1. Equation (3) is replaced by (Theorem 1.1 and Theorem 1.3)

$$
\sum_{\substack{q_{a^{\prime}} \leq x \\ \omega\left(q_{a^{\prime}}\right) \equiv 0(\bmod 2)}} 1=\frac{1}{2} \frac{6}{\pi^{2}} \frac{1}{a^{\prime \prime}} x+o(x) .
$$

If $a=1$ we put $a^{\prime}=a^{\prime \prime}=1$. The proof of (12) is by difference using (11) and Theorem 2.1 or using the equation

$$
\sum_{\substack{q_{a^{\prime}} \leq x \\ \omega\left(q_{a^{\prime}}\right) \equiv 1(\bmod 2)}} 1=\frac{1}{2} \frac{6}{\pi^{2}} \frac{1}{a^{\prime \prime}} x+o(x) .
$$

The theorem is proved.

Let $\Omega_{h, r}(x)$ be the number of $h$-full numbers $n_{h}$ not exceeding $x$ such that $\Omega\left(n_{h}\right) \equiv r(\bmod h)$ $(r=0, \ldots, h-1)$. We have the following theorem.

Theorem 2.4. The following asymptotic formulas hold.

$$
\Omega_{h, r}(x)=\frac{6}{\pi^{2}} C_{0, h, r} x^{\frac{1}{h}}+o\left(x^{\frac{1}{h}}\right) \quad(r=0, \ldots, h-1),
$$

where the constants $C_{0, h, r}$ are given by the series

$$
C_{0, h, r}=\sum_{\Omega(n) \equiv r(\bmod h)} \frac{1}{w(n)} \frac{1}{n^{\frac{1}{h}}} \quad(r=0, \ldots, h-1)
$$

and

$$
\sum_{r=0}^{h-1} C_{0, h, r}=C_{0, h}
$$

Proof. Since the total number of prime factors in the $h$-kernel is multiple of $h$, the proof is the same as the proof of Theorem 2.1, where we consider only the $h$-remainder $a$ such that $\Omega(a) \equiv r$ $(\bmod h)$. If $a=1$ we put $a^{\prime}=a^{\prime \prime}=1$ and $\Omega(a)=\Omega(1)=0$, therefore $\Omega(1) \equiv 0(\bmod h)$. The theorem is proved. 
Let $\Omega_{p, h}(x)$ be the number of $h$-full numbers $n_{h}$ not exceeding $x$ such that $\Omega\left(n_{h}\right)$ is even and let $\Omega_{i, h}(x)$ be the number of $h$-full numbers $n_{h}$ not exceeding $x$ such that $\Omega\left(n_{h}\right)$ is odd. We have the following theorem.

Theorem 2.5. If $h$ is even, then

$$
\begin{aligned}
& \Omega_{p, h}(x)=\frac{6}{\pi^{2}} D_{h, 0} x^{\frac{1}{h}}+o\left(x^{\frac{1}{h}}\right), \\
& \Omega_{i, h}(x)=\frac{6}{\pi^{2}} D_{h, 1} x^{\frac{1}{h}}+o\left(x^{\frac{1}{h}}\right),
\end{aligned}
$$

where the constants are given by the series

$$
\begin{gathered}
D_{h, 0}=\sum_{\Omega(n) \equiv 0(\bmod 2)} \frac{1}{w(n)} \frac{1}{n^{\frac{1}{h}}}=1+\sum_{n>1, \Omega(n) \equiv 0} \frac{1}{w(n)} \frac{1}{n^{\frac{1}{h}}}, \\
D_{h, 1}=\sum_{\Omega(n) \equiv 1(\bmod 2)} \frac{1}{w(n)} \frac{1}{n^{\frac{1}{h}}},
\end{gathered}
$$

and

$$
D_{h, 0}+D_{h, 1}=C_{0, h}
$$

If $h$ is odd, then

$$
\begin{aligned}
& \Omega_{p, h}(x)=\frac{1}{2} \frac{6}{\pi^{2}} C_{0, h} x^{\frac{1}{h}}+o\left(x^{\frac{1}{h}}\right), \\
& \Omega_{i, h}(x)=\frac{1}{2} \frac{6}{\pi^{2}} C_{0, h} x^{\frac{1}{h}}+o\left(x^{\frac{1}{h}}\right),
\end{aligned}
$$

Proof. If $h$ is even, then the total number of prime factors in the $h$-kernel is even, therefore, the proof is the same as the proof of Theorem 2.4. If $h$ is odd, in the proof of equation (13) we consider two cases.

Case 1. $\omega\left(q_{a^{\prime}}\right) \equiv 0(\bmod 2)$ and $\Omega(a) \equiv 0(\bmod 2)$.

Case $2 . \omega\left(q_{a^{\prime}}\right) \equiv 1(\bmod 2)$ and $\Omega(a) \equiv 1(\bmod 2)$.

Hence, the theorem is proved.

If $h=2$ (square-full numbers), we shall prove in the next theorem that $D_{2,0}>D_{2,1}$ and consequently the proportion of square-full numbers not exceeding $x$ with a total even number of prime factors is greater than the proportion of square-full numbers not exceeding $x$ with a total odd number of prime factors.

Theorem 2.6. The following inequality holds.

$$
D_{2,0}>D_{2,1}
$$


Proof. We have

$$
\sum_{n=1}^{\infty} \frac{1}{w(n)} \frac{1}{n}=\prod_{p}\left(1+\frac{1}{(p+1) p}+\frac{1}{(p+1) p^{2}}+\cdots\right)=\prod_{p}\left(\frac{1}{1-\frac{1}{p^{2}}}\right)=\frac{\pi^{2}}{6} .
$$

Let us consider the pairs $(a, b):(1,1),(2,3),(2,5),(2,7),(3,5),(2,11),(3,7),(2,13)$.

Note that by Remark 2.2 we have

$$
\frac{6}{\pi^{2}} D_{2,0}+\frac{6}{\pi^{2}} D_{2,1}=\frac{6}{\pi^{2}} C_{0,2}=2.1732543125 \ldots
$$

Now (see (16))

$$
\begin{aligned}
& \frac{6}{\pi^{2}} D_{2,0}>\frac{6}{\pi^{2}} \sum_{(a, b)}\left(\sum_{n=1}^{\infty} \frac{1}{w\left(a b n^{2}\right)} \frac{1}{\sqrt{a b n^{2}}}\right) \\
> & \left(\frac{6}{\pi^{2}} \sum_{n=1}^{\infty} \frac{1}{w(n)} \frac{1}{n}\right)\left(1+\sum_{(a, b) \neq(1,1)} \frac{1}{(a+1)(b+1)} \frac{1}{\sqrt{a b}}\right) \\
> & \frac{1}{2} \frac{6}{\pi^{2}} C_{0,2}=1.086627 \ldots
\end{aligned}
$$

since by (15) we have

$$
\frac{6}{\pi^{2}} \sum_{n=1}^{\infty} \frac{1}{w(n)} \frac{1}{n}=1
$$

Therefore (14) holds. The theorem is proved.

\section{Conclusion}

In this article we have studied the distribution of $h$-full numbers by use of an elementary method. By use of the same elementary method we have proved theorems on the functions $\omega(n)$ and $\Omega(n)$ defined on the sequence of $h$-full numbers. In particular, if $h=2$ then we have obtained that the square-full numbers with $\Omega(n)$ even are in greater proportion than the square-full numbers with $\Omega(n)$ odd.

\section{Acknowledgements}

The author is very grateful to Universidad Nacional de Luján.

\section{References}

[1] Bateman, P. T., \& Grosswald, E. (1958). On a theorem of Erdős and Szekeres, Illinois J. Math., 2, 88-98. 
[2] Djamel, B., Abdelmadjid, B., \& Ōzer, Ō.(2019). On a sequence formed by iterating a divisor operator, Czechoslovak Mathematical Journal, 69, 1177-1196 .

[3] Erdős, P., \& Szekeres, G. (1934-1935). Über die Anzahl der Abelschen Gruppen gegebener Ordnung und über ein verwandtes zahlentheoretisches Problem, Acta Sci. Math. (Szeged), 7, 95-102.

[4] Finch, S. (2003). Mathematical Constants, Cambridge University Press.

[5] Golomb, S. W. (1970). Powerful numbers, American Mathematical Monthly, 77, 848-852.

[6] Hardy, G. H., \& Ramanujan, S. (1917). The normal number of prime factors of a number $n$, Quart. J. Math., 48, 76-92.

[7] Hardy, G. H., \& Wright, E. M (1960). An Introduction to the Theory of Numbers, Oxford.

[8] Ivić, A. (2003). The Riemann Zeta-Function, Dover.

[9] Ivić, A., \& Shiu, P. (1982). The distribution of powerful numbers, Illinois J. Math., 26, 576-690.

[10] Jakimczuk, R. (2018). On the function $\omega(n)$, International Mathematical Forum, 13, 107-116.

[11] Jakimczuk, R. (2017). On the distribution of certain subsets of quadratfrei numbers, International Mathematical Forum, 12, 185-194.

[12] Jakimczuk, R.(2017). The kernel of powerful numbers, International Mathematical Forum $12,721-730$. 